\title{
Study of Energy Measurement of Cosmic Ray Nuclei with
}

\section{LHAASO}

\author{
Lu Bai ${ }^{a}$ and Hu Liu ${ }^{a, *}$ on behalf of the LHAASO Collaboration \\ (a complete list of authors can be found at the end of the proceedings) \\ ${ }^{a}$ Southwest Jiaotong University, \\ Xipu Campus, Southwest Jiaotong University, Chengdu, P. R. China, 611756 \\ E-mail: huliu@swjtu.edu.cn
}

The Large High Altitude Air Shower Observatory (LHAASO) is a hybrid extensive air shower (EAS) array with an area of about $1 \mathrm{~km}^{2}$ at an altitude of $4410 \mathrm{~m}$ a.s.l. in Sichuan province, China. It contains three sub-detectors: $1 \mathrm{~km}^{2}$ array (KM2A) composed of 5195 electromagnetic particle detector (ED) and muon detector (MD); water Cerenkov detector array (WCDA) and 18 Wide Field-of-view Cerenkov Telescope Array (WFCTA). One of the main scientific goals is measuring the individual energy spectra of cosmic rays from $\sim 30 \mathrm{TeV}$ to a couple of EeV. The construction of the whole observatory will be completed by the end of 2021. In this paper, a new energy reconstruction method and result based on KM2A simulated nuclei events will be shown.

$37^{\text {th }}$ International Cosmic Ray Conference (ICRC 2021)

July 12th - 23rd, 2021

Online - Berlin, Germany

\footnotetext{
${ }^{*}$ Presenter
} 


\section{Introduction}

The measurement of energy spectra of cosmic rays is an important tool to understand the origin, acceleration and propagation of cosmic ray [1,2]. The energy measurement is one of the main components for the spectra measurement. Large High Altitude Air Shower Observatory (LHAASO), located in Haizishan Daocheng, Sichuan province of China, is a hybrid experiment to detect cosmic rays [3]. The height of LHAASO is $4410 \mathrm{~m}$ above sea level. LHAASO has 18 Wide Field-of-View Cerenkov Telescopes (WFCTA), 78,000 $\mathrm{m}^{2}$ water Cerenkov detector array (WCDA), and $1 \mathrm{~km}^{2}$ Array (KM2A) including 5195 electromagnetic detectors (ED) and 1188 muon detectors (MD). LHAASO starts it's operation since April 2019, the construction of the whole observatory will be completed by the end of 2021. WFCTA, WCDA and KM2A measure the energy of primary particles with different techniques and independently. In this paper, a new energy reconstruction method and result from the measurements of KM2A detector will be shown.

\section{Experiment}

The central part of KM2A-EDs composed of 4901 EDs $\left(1 \mathrm{~m}^{2}\right.$ each) deployed in a grid with a spacing of $15 \mathrm{~m}$ to cover a circular area with a radius of $575 \mathrm{~m}$. This central part is surrounded by an outer guard-ring instrumented with 294 EDs (30 m spacing) up to a radius of 635m, mainly to improve the identification and the reconstruction of showers with the core outside the instrumented area. Each ED will be covered by a $0.5 \mathrm{~cm}$ thick lead plate ( 1 radiation length) to improve the angular resolution and to lower the energy threshold exploiting the pair production of secondary photons. The measured time resolution of a typical ED module is less than $2 \mathrm{~ns}$. To accomplish the physics program of KM2A, the EDs have been optimised to measure a wide range of particle densities, from $1 / m^{2}$ to $\sim 10,000 / m^{2}$ [4].

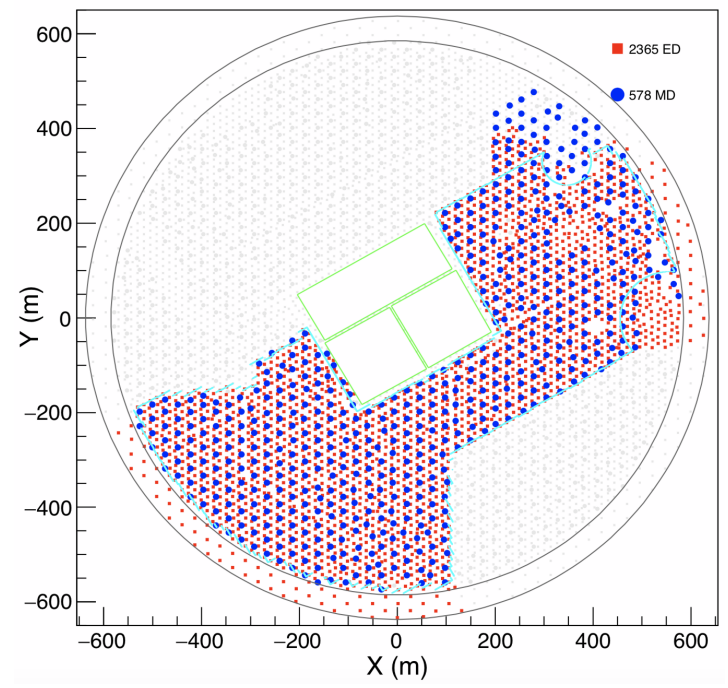

Figure 1: Planned layout of all LHAASO-KM2A detectors. The red squares and blue circles indicate the EDs and MDs of KM2A half-array

The KM2A-MD is composed of 1188 water Cerenkov tanks ( $36 \mathrm{~m}^{2}$ each) deployed in a grid with a spacing of $30 \mathrm{~m}$. The detectors are buried under $2.5 \mathrm{~m}$ of soil ( 12 radiation lengths) to 
reduce the punch-through due to shower electromagnetic particles. Therefore, the muon energy threshold is $1.3 \mathrm{GeV}$. The measured time resolution is about $10 \mathrm{~ns}$. The overall signal charge resolution for vertical single muon signals is estimated to be about $25 \%$ [4].

The KM2A half-array consists of 2365 EDs and 578 MDs, and takes data from Dec. 2019 to Dec. 2020. Planned layout of all LHAASO-KM2A detectors and the KM2A half-array are shown in Figure 1. The energy reconstruction in this paper is based on the KM2A half-array detectors.

\section{Monte Carlo simulation}

The simulations performed include the detailed air shower development in the atmosphere, as well as the response of the KM2A detector. Air showers were simulated in a wide energy range from $10^{13}$ to $10^{16} \mathrm{eV}$ with CORSIKA (v76400) [5]. The QGSJETII models for high-energy and FLUKA for low-energy hadronic interactions were used, and the EGS4 model was used for electromagnetic interactions. Five components-protons, helium, $\mathrm{CNO}, \mathrm{MgAlSi}$, and iron are generated in the above mentioned energy range, the mass number (noted as A below) for the CNO and MgAlSi groups is 14 and 27, respectively. Showers following an $E^{-1}$ energy spectrum and an isotropic angular distribution are simulated with a zenith angle range of 20-40 degrees and an azimuth range of 0-180 degrees, to fit the WFCTA field of view. The secondary particles reaching ground level are treated in a delicately developed detector simulation program in the framework of Geant4 package (named G4KM2A) [6]. The shower core position is evenly distributed in a circle with the center of the KM2A half-array as the origin and a radius of 1000 meters.

\section{Method}

Event reconstruction: The KM2A-ED detectors record the arriving time and charge of the secondary particles. The direction of primary particle is reconstructed by fitting the relative arriving time of ED hits. By combining the reconstructed direction of primary particle and information of the position and charge measurement of each ED detector, the lateral distribution of secondary charged particles (number density versus the perpendicular distance to the shower axis) is obtained. The core position, shower size (the integration of lateral distribution over area, noted as Nsize below) and age parameter are reconstructed by fitting the lateral distribution with a modified NishimuraKamata-Greisen (NKG) function using a likelihood method [7]. During the reconstruction, only the ED hits within the time and space window are used. Detailed information about the reconstruction can be found in article [4].

The KM2A-MD detectors record the charge of muons, to reduce pollution from the punchthrough high energy electrons/positrons near the shower core, only MD detectors with a distance further than 40 meters from the core are used to obtain the number of muons (noted as $\mathrm{N} \mu$ below).

The Nsize from ED measurement and the $\mathrm{N} \mu$ from MD measurement are used to reconstruct energy and do particle identification between nuclei for the Monte Carlo samples. Both Nsize and $\mathrm{N} \mu$ are good energy estimators, since they are proportional to the energy of primary particle. However, they are also affected by other variables. For example, the zenith angle of primary particle that changes the integrated mass of atmosphere traversed, the age parameter from the fitting of lateral distribution that reflects the stage of EAS development, which is related to Xmax information of the 
longitudinal development of the shower [7], and also the mass of the primary particle. In this paper, the age parameter is used to correct for shower fluctuation, and the zenith angle is used to correct for different atmosphere mass. Those two effects are corrected before the energy reconstruction. The effects of the type of primary particle (or different mass) is also corrected by combining the energy measurements of Nsize and $\mathrm{N} \mu$ in the end.
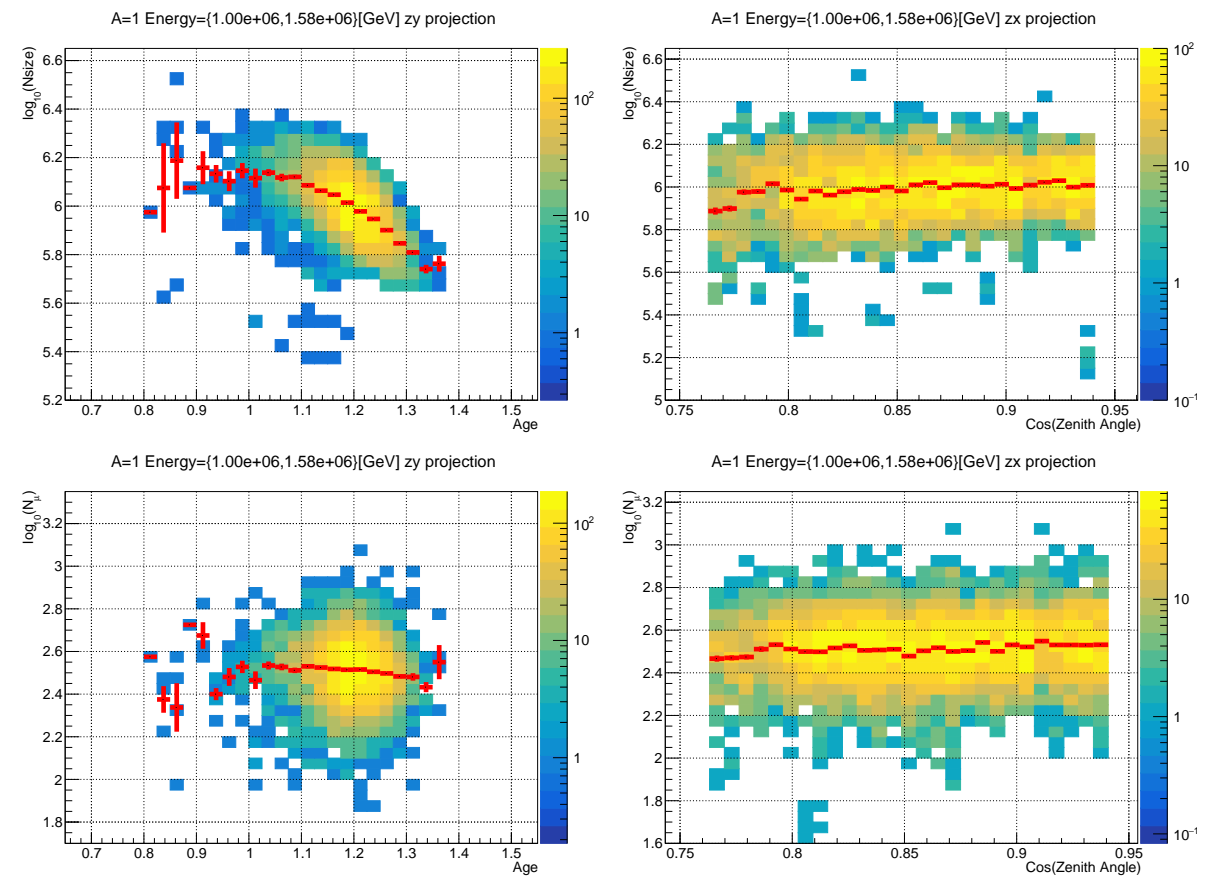

Figure 2: The dependences of Nsize and $\mathrm{N} \mu$ on age and zenith angle of primary particle for proton MC samples. Upper left: Nsize vs age; upper right: Nsize vs $\cos (z e n i t h$ angle); lower left: $\mathrm{N} \mu$ vs age; lower right: $\mathrm{N} \mu$ vs $\cos (z e n i t h$ angle). The red lines correspond to the mean value.

To obtain the dependencies of Nsize and $\mathrm{N} \mu$ on age and zenith angle for different mass and different energy, the MC samples is used. The dependence of the Nsize and $\mathrm{N} \mu$ on age and zenith angle for proton samples with energy $\sim 1 \mathrm{PeV}$ are shown in Figure 2. As shown in Figure 2, the Nsize has a strong dependence on the age, mainly due to shower fluctuations, and small dependence on zenith angle. The dependencies of $\mathrm{N} \mu$ on age and zenith angle are both small. The dependencies for other energy ranges and other type of primary particles are also similar.

Energy reconstruction: Due to the unknown type of primary particle in real data, instead of correct the measured Nsize and $\mathrm{N} \mu$ to a value associated to a reference age and zenith angle. For a shower event with reconstructed age parameter and zenith angle, the mean Nsize and $\mathrm{N} \mu$ versus generated energy for different type of primary particles are reconstructed based on the dependencies above, which are shown in Figure $3 \mathrm{a}$ and $4 \mathrm{a}$, when age $=1.3$ and zenith angle $=30^{\circ}$. For a giving Nsize (or $\mathrm{N} \mu$ ), based on the lines shown in Figure 3a and 4a, one energy number is reconstructed for each type (or mass) of primary particle. Figure $3 \mathrm{~b}$ and $4 \mathrm{~b}$ show the reconstructed energy versus true logarithm mass $\log _{10}$ (A) for an event with Nsize $=10^{6.0}$ and $\mathrm{N} \mu=10^{2.7}$. As shown, the reconstructed 


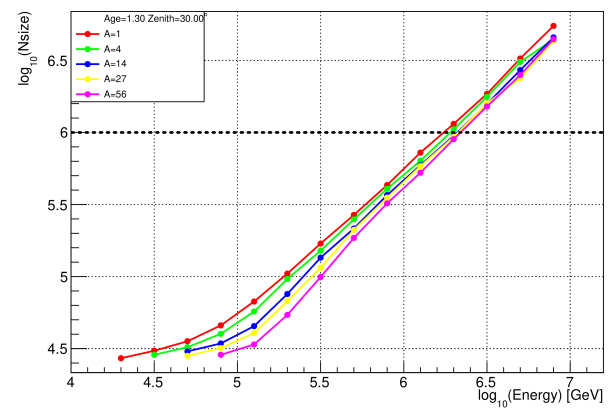

(a)

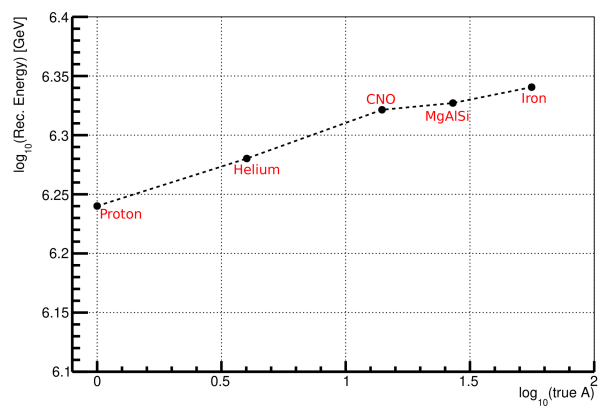

(b)

Figure 3: (a): The Nsize versus the true energy for different mass samples of MC (different colors), the dashed line indicate Nsize $=10^{6.0}$; (b): The reconstructed energy from Nsize versus the true logarithm mass of primary particle for an event with Nsize $=10^{6.0}$, based on the correlations between Nsize and true energy in (a). In both plots, age $=1.3$ and zenith angle $=30^{\circ}$.

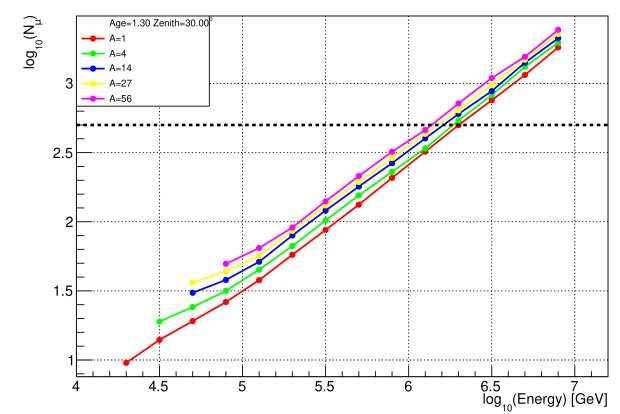

(a)

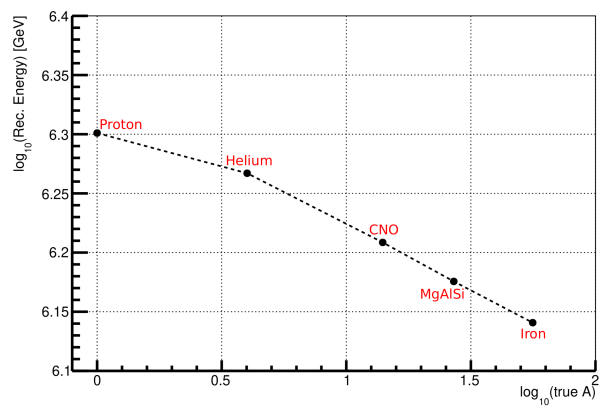

(b)

Figure 4: (a): The $\mathrm{N} \mu$ versus the true energy for different mass samples of MC (different colors), the dashed line indicate $\mathrm{N} \mu=10^{2.7}$; (b): The reconstructed energy from $\mathrm{N} \mu$ versus the true logarithm mass of primary particle for an event with $\mathrm{N} \mu=10^{2.7}$, based on the correlations between $\mathrm{N} \mu$ and true energy in (a). In both plots, age $=1.3$ and zenith angle $=30^{\circ}$.

energy is also depending on the mass of primary particle, and the dependencies are different between Nsize and $\mathrm{N} \mu$, since they are energy estimators based on different kinds of secondaries. However, the energy and mass of the primary particle for this event should follow both lines in Figure $3 \mathrm{~b}$ and $4 \mathrm{~b}$. Then the mass and energy should be the $\mathrm{x}$ and $\mathrm{y}$ coordinate of the cross point of the two dashed lines in Figure $3 \mathrm{~b}$ and $4 \mathrm{~b}$. In this way, the energy and A are reconstructed simultaneously by combining the Nsize and $\mathrm{N} \mu$ measurements, and during this procedure, the mass dependence of the primary particle is also corrected. These procedures are repeated in the parameter spaces (four parameters: Nsize, $\mathrm{N} \mu$, age and zenith angle) to reconstruct the energy and A. Since the energy and A reconstruction are done for each age and zenith angle, all the dependencies of Nsize and $\mathrm{N} \mu$ on age and zenith angle are corrected automatically. And the dependency on the mass of primary particle is also counted by combining the two independent energy measurements from Nsize and $\mathrm{N} \mu$. 


\section{Result}
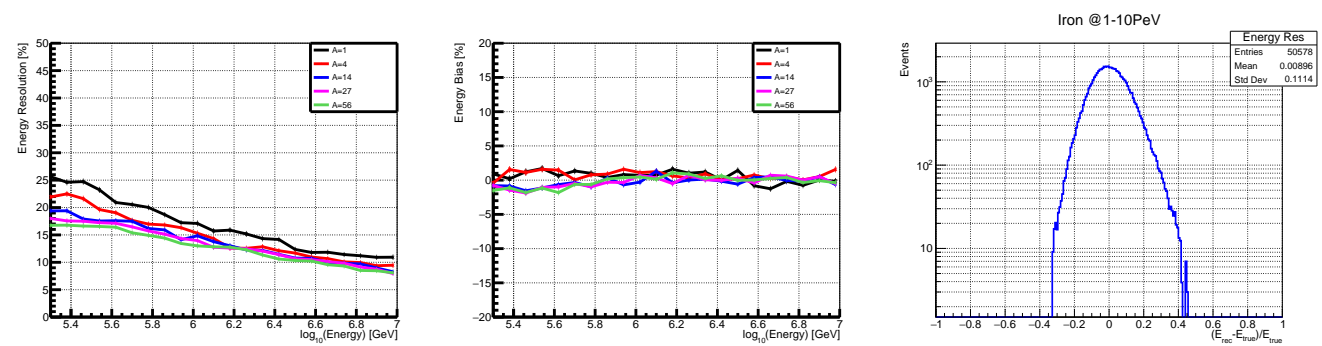

Figure 5: The parameters of the energy reconstruction method in the enegry range from $300 \mathrm{TeV}$ to $10 \mathrm{PeV}$. Left: the energy resolution in percentage for five mass groups; middle: the energy bias in percentage for five mass groups; right: the energy resolution function in the energy range from $1 \mathrm{PeV}$ to $10 \mathrm{PeV}$ of iron sample.

The energy resolution and energy bias for proton, helium, $\mathrm{CNO}, \mathrm{MgAlSi}$ and iron $\mathrm{MC}$ samples in the energy range from $300 \mathrm{TeV}$ to $10 \mathrm{PeV}$ are shown in Figure 5. Left panel of Figure 5 shows the energy resolution in percentage, middle panel of Figure 5 shows the energy bias in percentage. In the right panel of Figure 5, the energy resolution function in the energy range from $1 \mathrm{PeV}$ to 10 $\mathrm{PeV}$ of iron sample is also shown.

\section{Acknowledgements}

This work is supported in China by the Fundamental Research Funds for the Central Universities (grant numbers 2682020CX77, 2682020CX73, 2682020CX74). It is also supported by the Science and Technology Department of Sichuan Province (grant numbers 2021YFSY0031, 2020YFSY0016), and by NSFC (grant number 11947404), and by National Key R\&D program of China (grant number 2018YFA0404201).

\section{References}

[1] B. Bartoli et al., PHYSICAL REVIEW D 92, 092005 (2015)

[2] T. Antoni et al., Astrop. Physics 24, 1 (2005); W.D. Apel et al., Astrop. Physics 31, 86 (2009); W.D. Apel et al., Astrop. Physics 47, 54 (2013)

[3] Z. Cao et al., Chinese Physics C 34, 249 (2010)

[4] F. Aharonian et al., Chin. Phys. C 45, 025002 (2021)

[5] D. Heck et al., CORSIKA: a Monte Carlo code to simulate extensive air showers. (1998)

[6] S. Agostinelli et al., Nucl. Instrum. Methods Phys. Res., Sect. A 506, 250 (2003)

[7] B. Bartoli et al., Astrop. Physics 93, 46 (2017); 


\section{Full Authors List: LHAASO Collaboration}

Zhen $\mathrm{Cao}^{1,2,3}$, F. Aharonian ${ }^{4,5}$, Q. An ${ }^{6,7}$, Axikegu ${ }^{8}$, L.X. Bai ${ }^{9}$, Y.X. Bai ${ }^{1,3}$, L.X. Bai ${ }^{9}$, Y.X. Bai ${ }^{1,3}$, Y.W. Bao ${ }^{10}$, D. Bastieri ${ }^{11}$, X.J. Bi ${ }^{1,2,3}$, Y.J. Bi ${ }^{1,3}$, H. Cai ${ }^{12}$, J.T. Cai ${ }^{11}$, Zhe Cao ${ }^{6,7}$, J. Chang ${ }^{13}$, J.F. Chang ${ }^{1,3,6}$, B.M. Chen ${ }^{14}$, E.S. Chen ${ }^{1,2,3}$, J. Chen ${ }^{9}$, Liang Chen $^{1,2,3}$, Liang Chen ${ }^{15}$, Long Chen ${ }^{8}$, M.J. Chen ${ }^{1,3}$, M.L. Chen ${ }^{1,3,6}$, Q.H. Chen ${ }^{8}$, S.H. Chen ${ }^{1,2,3}$, S.Z. Chen ${ }^{1,3}$, T.L. Chen ${ }^{16}$,X.L. $\mathrm{Chen}^{1,2,3}$, Y. Chen $^{10}$, N. Cheng ${ }^{1,3}$, Y.D. Cheng ${ }^{1,3}$, S.W. Cui ${ }^{14}$, X.H. Cuii ${ }^{17}$, Y.D. Cui ${ }^{18}$, B. D'Ettorre Piazzoli ${ }^{19}$, B.Z. Dai ${ }^{20}$, H.L.

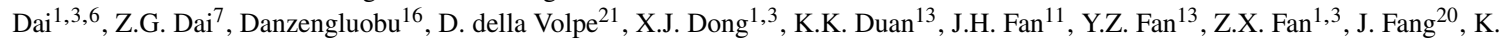
Fang $^{1,3}$, C.F. Feng ${ }^{22}$, L. Feng ${ }^{13}$, S.H. Feng ${ }^{1,3}$, Y.L. Feng ${ }^{13}$, B. Gao ${ }^{1,3}$, C.D. Gao ${ }^{22}$, L.Q. Gao ${ }^{1,2,3}$, Q. Gao ${ }^{16}$, W. Gao ${ }^{22}$, M.M. Ge ${ }^{20}$, L.S. Geng ${ }^{1,3}$, G.H. Gong ${ }^{23}$, Q.B. Gou ${ }^{1,3}$, M.H. Gu${ }^{1,3,6}$, F.L. Guo ${ }^{15}$, J.G. Guo ${ }^{1,2,3}$, X.L. Guo ${ }^{8}$, Y.Q. Guo ${ }^{1,3}$, Y.Y. Guo ${ }^{1,2,3,13}$, Y.A. $\mathrm{Han}^{24}$, H.H. He $\mathrm{H}^{1,2,3}$, H.N. He ${ }^{13}$, J.C. He ${ }^{1,2,3}$, S.L. He ${ }^{11}$, X.B. He ${ }^{18}$, Y. He ${ }^{8}$, M. Heller ${ }^{21}$, Y.K. Hor ${ }^{18}$, C. Hou ${ }^{1,3}$, H.B. Hu ${ }^{1,2,3}$, S.

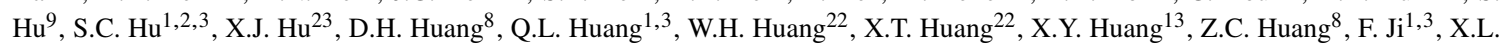
$\mathrm{Ji}^{1,3,6}$, H.Y. Jia ${ }^{8}$, K. Jiang ${ }^{6,7}$, Z.J. Jiang ${ }^{20}$, C. Jin ${ }^{1,2,3}$, T. Ke ${ }^{1,3}$, D. Kuleshov ${ }^{25}$, K. Levochkin ${ }^{25}$, B.B. Li ${ }^{14}$, Cheng Li ${ }^{6,7}$, Cong Li ${ }^{1,3}$,

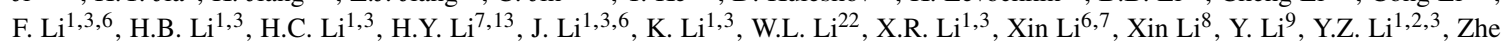
$\mathrm{Li}^{1,3}$, Zhuo Li ${ }^{26}$, E.W. Liang ${ }^{27}$, Y.F. Liang ${ }^{27}$, S.J. Lin ${ }^{18}$, B. Liu ${ }^{7}$, C. Liu ${ }^{1,3}$, D. Liu ${ }^{22}$, H. Liu ${ }^{8}$, H.D. Liu ${ }^{24}$, J. Liu ${ }^{1,3}$, J.L. Liu ${ }^{28}$, J.S.

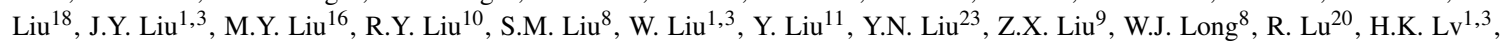

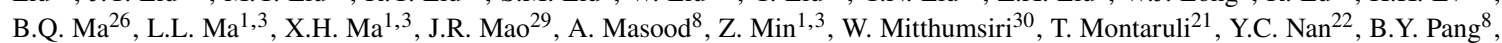
P. Pattarakijwanich ${ }^{30}$, Z.Y. Pei ${ }^{11}$, M.Y. Qi ${ }^{1,3}$, Y.Q. Qi ${ }^{14}$, B.Q. Qiao ${ }^{1,3}$, J.J. Qin ${ }^{7}$, D. Ruffolo ${ }^{30}$, V. Rulev ${ }^{25}$, A. Sáiz ${ }^{30}$, L. Shao ${ }^{14}$, O. Shchegolev $^{25,31}$, X.D. Sheng ${ }^{1,3}$, J.Y. Shi ${ }^{1,3}$, H.C. Song ${ }^{26}$, Yu.V. Stenkin ${ }^{25,31}$, V. Stepanov ${ }^{25}$, Y. Su ${ }^{32}$, Q.N. Sun ${ }^{8}$, X.N. Sun ${ }^{27}$, Z.B.

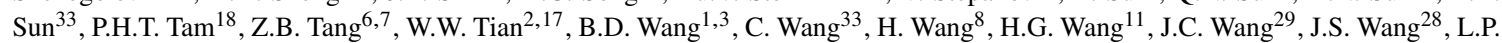
Wang $^{22}$, L.Y. Wang ${ }^{1,3}$, R.N. Wang ${ }^{8}$, W. Wang ${ }^{18}$, W. Wang ${ }^{12}$, X.G. Wang ${ }^{27}$, X.J. Wang ${ }^{1,3}$, X.Y. Wang ${ }^{10}$, Y. Wang ${ }^{8}$, Y.D. Wang ${ }^{1,3}$, Y.J. Wang $^{1,3}$, Y.P. Wang ${ }^{1,2,3}$, Z.H. Wang ${ }^{9}$, Z.X. Wang ${ }^{20}$, Zhen Wang ${ }^{28}$, Zheng Wang ${ }^{1,3,6}$, D.M. Wei ${ }^{13}$, J.J. Wei ${ }^{13}$, Y.J. Wei ${ }^{1,2,3}$, T. Wen $^{20}$, C.Y. Wu ${ }^{1,3}$, H.R. Wu ${ }^{1,3}$, S. $\mathrm{Wu}^{1,3}$, W.X. Wu ${ }^{8}$, X.F. Wu ${ }^{13}$, S.Q. Xi ${ }^{1,3}$, J. Xia ${ }^{7}, 13$, J.J. Xia ${ }^{8}$, G.M. Xiang ${ }^{2,15}$, D.X. Xiao ${ }^{16}$, G. Xiao ${ }^{1,3}$, H.B. Xiao ${ }^{11}$, G.G. Xin ${ }^{12}$, Y.L. Xin ${ }^{8}$, Y. Xing ${ }^{15}$, D.L. Xu ${ }^{28}$, R.X. Xu ${ }^{26}$, L. Xue ${ }^{22}$, D.H. Yan ${ }^{29}$, J.Z. Yan ${ }^{13}$, C.W. Yang 9 , F.F. Yang ${ }^{1,3,6}$, J.Y. Yang ${ }^{18}$, L.L. Yang ${ }^{18}$, M.J. Yang ${ }^{1,3}$, R.Z. Yang ${ }^{7}$, S.B. Yang ${ }^{20}$, Y.H. Yao ${ }^{9}$, Z.G. Yao ${ }^{1,3}$, Y.M. Ye ${ }^{23}$, L.Q. Yin ${ }^{1,3}$, N. Yin ${ }^{22}$, X.H. You $^{1,3}$, Z.Y. You ${ }^{1,2,3}$, Y.H. Yu ${ }^{22}$, Q. Yuan ${ }^{13}$, H.D. Zeng ${ }^{13}$, T.X. Zeng ${ }^{1,3,6}$, W. Zeng ${ }^{20}$, Z.K. Zeng ${ }^{1,2,3}$, M. Zha ${ }^{1,3}$, X.X. Zhai ${ }^{1,3}$, B.B. Zhang $^{10}$, H.M. Zhang ${ }^{10}$, H.Y. Zhang ${ }^{22}$, J.L. Zhang ${ }^{17}$, J.W. Zhang ${ }^{9}$, L.X. Zhang ${ }^{11}$, Li Zhang ${ }^{20}$, Lu Zhang ${ }^{14}$, P.F. Zhang ${ }^{20}$, P.P. Zhang ${ }^{14}$, R. Zhang ${ }^{7,13}$, S.R. Zhang ${ }^{14}$, S.S. Zhang ${ }^{1,3}$, X. Zhang ${ }^{10}$, X.P. Zhang ${ }^{1,3}$, Y.F. Zhang ${ }^{8}$, Y.L. Zhang ${ }^{1,3}$, Yi Zhang ${ }^{1,13}$, Yong Zhang ${ }^{1,3}$, B. $Z_{\text {Zhao }}^{8}$, J. Zhao ${ }^{1,3}$, L. Zhao ${ }^{6,7}$, L.Z. Zhao ${ }^{14}$, S.P. Zhao ${ }^{13,22}$, F. Zheng ${ }^{33}$, Y. Zheng ${ }^{8}$, B. Zhou ${ }^{1,3}$, H. Zhou ${ }^{28}$, J.N. Zhou ${ }^{15}$, P. Zhou ${ }^{10}$, R. Zhou $^{9}$, X.X. Zhou ${ }^{8}$, C.G. Zhu ${ }^{22}$, F.R. Zhu ${ }^{8}$, H. Zhu ${ }^{17}$, K.J. Zhu ${ }^{1,2,3,6}$ and X. Zuo ${ }^{1,3}$

${ }^{1}$ Key Laboratory of Particle Astrophyics \& Experimental Physics Division \& Computing Center, Institute of High Energy Physics, Chinese Academy of Sciences, 100049 Beijing, China.

${ }^{2}$ University of Chinese Academy of Sciences, 100049 Beijing, China.

${ }^{3}$ TIANFU Cosmic Ray Research Center, Chengdu, Sichuan, China.

${ }^{4}$ Dublin Institute for Advanced Studies, 31 Fitzwilliam Place, 2 Dublin, Ireland.

${ }^{5}$ Max-Planck-Institut for Nuclear Physics, P.O. Box 103980, 69029 Heidelberg, Germany.

${ }^{6}$ State Key Laboratory of Particle Detection and Electronics, China.

${ }^{7}$ University of Science and Technology of China, 230026 Hefei, Anhui, China.

${ }^{8}$ School of Physical Science and Technology \& School of Information Science and Technology, Southwest Jiaotong University, 610031 Chengdu, Sichuan, China.

${ }^{9}$ College of Physics, Sichuan University, 610065 Chengdu, Sichuan, China.

${ }^{10}$ School of Astronomy and Space Science, Nanjing University, 210023 Nanjing, Jiangsu, China.

${ }^{11}$ Center for Astrophysics, Guangzhou University, 510006 Guangzhou, Guangdong, China.

${ }^{12}$ School of Physics and Technology, Wuhan University, 430072 Wuhan, Hubei, China.

${ }^{13}$ Key Laboratory of Dark Matter and Space Astronomy, Purple Mountain Observatory, Chinese Academy of Sciences, 210023 Nanjing, Jiangsu, China.

${ }^{14}$ Hebei Normal University, 050024 Shijiazhuang, Hebei, China.

${ }^{15}$ Key Laboratory for Research in Galaxies and Cosmology, Shanghai Astronomical Observatory, Chinese Academy of Sciences, 200030 Shanghai, China.

${ }^{16}$ Key Laboratory of Cosmic Rays (Tibet University), Ministry of Education, 850000 Lhasa, Tibet, China.

${ }^{17}$ National Astronomical Observatories, Chinese Academy of Sciences, 100101 Beijing, China.

${ }^{18}$ School of Physics and Astronomy \& School of Physics (Guangzhou), Sun Yat-sen University, 519000 Zhuhai, Guangdong, China.

${ }^{19}$ Dipartimento di Fisica dell'Università di Napoli 'Federico II', Complesso Universitario di Monte Sant’Angelo, via Cinthia, 80126 Napoli, Italy.

${ }^{20}$ School of Physics and Astronomy, Yunnan University, 650091 Kunming, Yunnan, China.

${ }^{21} \mathrm{D}_{i}{ }^{-}$epartement de Physique Nucl ${ }_{i}{ }^{-}$eaire et Corpusculaire, Facult ${ }_{i}^{-}$e de Sciences, Universiti ${ }^{-}$e de Gen 'eve, 24 Quai Ernest Ansermet, 1211 Geneva, Switzerland.

${ }^{22}$ Institute of Frontier and Interdisciplinary Science, Shandong University, 266237 Qingdao, Shandong, China.

${ }^{23}$ Department of Engineering Physics, Tsinghua University, 100084 Beijing, China.

${ }^{24}$ School of Physics and Microelectronics, Zhengzhou University, 450001 Zhengzhou, Henan, China.

${ }^{25}$ Institute for Nuclear Research of Russian Academy of Sciences, 117312 Moscow, Russia. 
${ }^{26}$ School of Physics, Peking University, 100871 Beijing, China.

${ }^{27}$ School of Physical Science and Technology, Guangxi University, 530004 Nanning, Guangxi, China.

${ }^{28}$ Tsung-Dao Lee Institute \& School of Physics and Astronomy, Shanghai Jiao Tong University, 200240 Shanghai, China.

${ }^{29}$ Yunnan Observatories, Chinese Academy of Sciences, 650216 Kunming, Yunnan, China.

${ }^{30}$ Department of Physics, Faculty of Science, Mahidol University, 10400 Bangkok, Thailand.

${ }^{31}$ Moscow Institute of Physics and Technology, 141700 Moscow, Russia.

${ }^{32}$ Key Laboratory of Radio Astronomy, Purple Mountain Observatory, Chinese Academy of Sciences, 210023 Nanjing, Jiangsu, China.

${ }^{33}$ National Space Science Center, Chinese Academy of Sciences, 100190 Beijing, China. 